\title{
Algebraic and analytic reconstruction methods for dynamic tomography.
}

\author{
L. Desbat, S. Rit, R. Clackdoyle, C. Mennessier, E. Promayon and S. Ntalampeki
}

\begin{abstract}
In this work, we discuss algebraic and analytic approaches for dynamic tomography. We present a framework of dynamic tomography for both algebraic and analytic approaches. We finally present numerical experiments.
\end{abstract}

\section{INTRODUCTION}

Dynamic tomography is a very active area [1], [2]. Movements or deformations of the patient must be taken into account in nuclear medicine, such as SPECT or PET because of long measurement time, but also in CT for fast moving organs such as the heart. Generally, patient movements or deformations occur in 3D. In this work we consider dynamic 3D Cone Beam tomography. We present both algebraic and analytic aproaches to compensate for the patient deformation during the reconstruction.

\section{A. 3D cone beam notations}

Let $f: \mathbb{R}^{3} \rightarrow \mathbb{R}$ be the 3D attenuation distribution to be reconstructed from projections (x-ray, SPECT or PET). In this work, we consider mainly the $3 \mathrm{D}$ cone beam transform

$$
g_{\mathcal{D}}(t, \vec{\zeta}) \stackrel{\text { def }}{=} \mathcal{D} f(t, \vec{\zeta}) \stackrel{\text { def }}{=} \mathcal{D}_{t} f(\vec{\zeta}) \stackrel{\text { def }}{=} \int_{0}^{+\infty} f(\vec{a}(t)+l \vec{\zeta}) d l,
$$

where $\vec{\zeta} \in S^{2}$ is a unit vector in $\mathbb{R}^{3}$ ( $S^{2}$ is the unit sphere in $\mathbb{R}^{3}$ ), $\vec{a}(t) \in \mathbb{R}^{3}$ is the x-ray source position at time $t \in T \subset \mathbb{R}, t$ is then also the source trajectory parameter in $\mathbb{R}^{3}$, see Fig. $1 . \mathcal{D}_{t} f(\vec{\zeta})$, at fixed $t$, is assumed to be acquired in a negligible time, $\forall \vec{\zeta} \in S^{2}$. The function $f$ is assumed to have a compact support and $\vec{a}(t)$ has a strictly positive distance to the support of $f$. This transform appears in 3D x-ray tomography (reconstruction from 2D xray projections or multiline $\mathrm{CT}$ ) with applications in cardiac $\mathrm{CT}$ or radiotherapy.

$3 \mathrm{D}$ cone beam tomography problem is the reconstruction of $f$ from $g_{\mathcal{D}}$. These last years, new developments have been proposed to solve analytically, exactly, and efficiently this problem, in particular for the helical source trajectory, but also for more general trajectory (see for exemple [3], [4], [5], [6], [7], [8]).

This work was supported by the ANR grant NT05-1_45428, ToRIID

L. Desbat, E. Promayon and S. Ntalampeki are with TIMC-IMAG, In3S, Medical Faculty, Grenoble University, 38000 Grenoble, France Laurent. Desbateimag. fr

S. Rit is with the LIRIS, Université Lumière Lyon 2, Lyon, France simon.ritegmail.com

R. Clackdoyle and C. Mennessier are with LHC, St Etienne, France rolf.clackdoyleduniv-st-etienne.fr, mennessier@cpe.fr

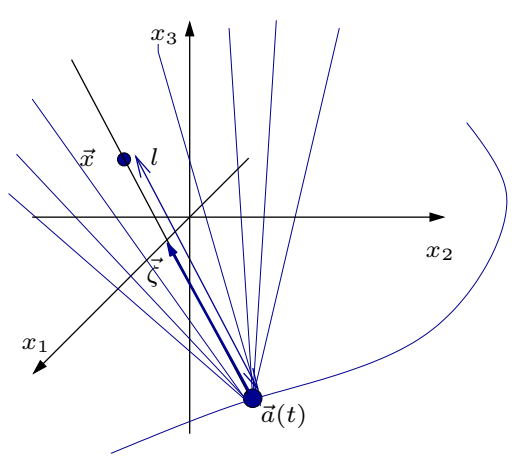

Fig. 1. Cone Beam geometry parameters

\section{B. Deformation model}

In dynamic tomography, the attenuation $f$ to be reconstructed is also a function of $t$. In our approach, we consider a time dependent deformation of the space as in [9], [10]. We introduce a time dependent deformation model $\vec{\Gamma}_{t}$ and we assume that, for $t \in T, \vec{\Gamma}_{t}$ are known bijective appropriately smooth functions on $\mathbb{R}^{3}$ whose inverse are smooth too. We assume that the attenuation at point $\vec{x} \in \mathbb{R}^{3}$ at time $t$ can be written in the form $f_{\vec{\Gamma}_{t}}(\vec{x})=f\left(\vec{\Gamma}_{t}(\vec{x})\right)$, where $f$ is the attenuation reference point (for example, at $t=0$ ). So $\vec{\Gamma}_{t}$ simply transforms the position $\vec{x}$ at time $t$ to its position $\vec{\Gamma}_{t}(\vec{x})$ at the reference time $(t=0)$.

The problem under consideration is the reconstruction of $f$, or equivalently of $f_{\vec{\Gamma}_{t}}$, from cone-beam measurements $\mathcal{D} f_{\vec{\Gamma}_{t}}$, see (1), for known deformation functions $\vec{\Gamma}_{t}, t \in T$. Our objective is to apply efficient accurate deformation corrections in the reconstruction algorithms.

\section{Dynamic reconstruction approaches}

General patient deformations can be nowadays compensated only by algebraic reconstruction approaches. Iterative reconstruction requires an adequate projector able to perform the attenuation sum (1) based on a discrete representation of $f$ at the reference time. This is done in the static case by computing the intersection of the straight acquisition lines with basis functions, e.g. voxels. In the dynamic case, the projector must compensate for the motion. If $\vec{\Gamma}_{t}$ preserves the rectitude of acquisition lines, i.e. if it transforms a set of convergent aquisition lines at time $t$ into a set of convergent lines at the reference time, this can be done as in the static case along the virtual transformed lines. In particular, if the patient motion is globally rigid, this amounts to using virtual source and detector positions and applying the reconstruction algorithm as in the static case [11], [12], [13] or to deform 
data prior to reconstruction [14], [15]. These approaches can be generalized to deformations transforming the set of acquisition lines of each cone beam projection into other sets of concurent lines [9], [10], [16]. Thus, analytic approaches essentially allow for the compensation of deformations in subclasses of those presented in section I-B. With this approach, deformations leaving globally invariant the cone beam geometry acquisition can be compensated. When this is not the case, iterative reconstruction is still feasible but the intersection of the straight acquisition line with $f_{\vec{\Gamma}_{t}}(\vec{x})$ should take into account the translation and the deformation of the basis functions. Moreover, in 3D interventional image reconstruction from $\mathrm{x}$-ray projections, the source trajectory is very often a circle [17], [18] for which exact analytic inversion does not apply whereas algebraic approaches are well defined. When a high contrast object like coronary arteries is being reconstructed, the deformation can be ignored [19], [20]. Otherwise, one has to find a way to deform the basis functions. For example, spherical basis functions can be deformed in ellipoids as a first approximation [21], [22].

In the next section, we present a dynamic tomography algebraic method framework. In section III, we present an analytic approach for dynamic tomography. Then, we present some numerical experiments in section IV.

\section{ALGEBRAIC DYNAMIC RECONSTRUCTION}

In algebraic approch, we assume that the unknown function $f$ can decomposed into a finite linear combination of given independent functions, such as voxel indicator functions. Let $\left(e_{j}\right)_{j \in J}$ be a set of basis functions $e_{j}: \mathbb{R}^{3} \rightarrow \mathbb{R}, J$ being a finite set of index such as $J=\left\{1, \ldots, n_{J}\right\}, n_{J} \in \mathbb{N}$ denoting the number of elements \# $(J)$ of the set $J$ (multi index sets are also very often used in multi dimension space). For example, $e_{j}$ can be the indicator function of the voxel number $j$ in the reconstruction region. We assume that

$$
f(\vec{x})=\sum_{j \in J} f_{j} e_{j}(\vec{x}),
$$

where $f_{j} \in \mathbb{R}, j \in J$. We also assume that the acquisition can be modeled by

$$
d_{i}=\int_{\Omega} h_{i}(\vec{x}) f(\vec{x}) d \vec{x}, i \in I .
$$

where $\left(d_{i}\right)_{i \in I}$ is the real vector of acquired data. In 2D tomography, $h_{i}(\vec{x})$ could be the dirac on a line $\delta\left(\vec{x} \cdot \vec{\theta}_{i}-s_{i}\right)$ where $\vec{\theta}_{i}$ is the direction of the projection and $s_{i} \in \mathbb{R}$ is the signed distance of the line to the center. In $3 \mathrm{D} h_{i}(\vec{x})$ could be the dirac on the x-ray line but it could also be the indicator of the conical region joining a point source $\vec{a}_{i}$ and a detector or some smooth response function obtained by calibration of the X-ray, PET or SPECT system. In nuclear imaging, $h_{i}$ can also model more physics such as attenuation, etc. The number of data $n_{I}$ is finite.
In static tomography, the algebraic aproach yields a linear system to be solved

$$
\begin{aligned}
d_{i} & =\int_{\Omega} h_{i}(\vec{x}) f(\vec{x}) d \vec{x}=\int_{\Omega} h_{i}(\vec{x}) \sum_{j \in J} f_{j} e_{j}(\vec{x}) d \vec{x} \\
& =\sum_{j \in J}\left(\int_{\Omega} h_{i}(\vec{x}) e_{j}(\vec{x}) d \vec{x}\right) f_{j}
\end{aligned}
$$

We have to solve the linear system $\mathbf{d}=\mathbf{A f}$ where $\mathbf{d}=$ $\left(d_{i}\right)_{i=1, \ldots, n_{I}}$ is the known vector of data, $\mathbf{f}=\left(f_{j}\right)_{j=1, \ldots, n_{J}}$ is the unknown vector of coefficients of $f$ to be identified and the matrix entry $A_{i, j}$ is $A_{i, j}=\int_{\Omega} h_{i}(\vec{x}) e_{j}(\vec{x}) d \vec{x}$. The matrix is generally sparse because both $h_{i}$ and $e_{j}$ functions have a limited support in the domain $\Omega \subset \mathbb{R}^{3}$. Thus iterative methods are used to solve the linear system.

In dynamic tomography

$$
\begin{aligned}
d_{t, i} & =\int_{\Omega} h_{i}(\vec{x}) f\left(\vec{\Gamma}_{t}(\vec{x})\right) d \vec{x} \\
& =\sum_{j \in J}\left(\int_{\Omega} h_{i}(\vec{x}) e_{j}\left(\vec{\Gamma}_{t}(\vec{x})\right) d \vec{x}\right) f_{j}
\end{aligned}
$$

Let us now assume that the functions $e_{j}\left(\vec{\Gamma}_{t}(\vec{x})\right)$ can be decomposed (or approximated) into a finite linear combination of given independent functions $\left(b_{k}\right)_{k \in K}, K$ being a finite index (or multi-index) set, more precisely

$$
e_{j}\left(\vec{\Gamma}_{t}(\vec{x})\right)=\sum_{k \in K} \Gamma_{t_{k, j}} b_{k}(\vec{x}) .
$$

then (6) introduced in (5) yields

$$
\begin{aligned}
d_{t, i} & =\sum_{j \in J}\left(\int_{\Omega} h_{i}(\vec{x}) \sum_{k \in K} \Gamma_{t_{k, j}} b_{k}(\vec{x}) d \vec{x}\right) f_{j} \\
& =\sum_{j \in J}\left(\sum_{k \in K} B_{i, k} \Gamma_{t_{k, j}}\right) f_{j}
\end{aligned}
$$

where $\mathbf{B}$ is the algebraic matrix for the basis function $\left(b_{k}\right)_{k \in K}$

$$
B_{i, k}=\int_{\Omega} h_{i}(\vec{x}) b_{k}(\vec{x}) d \vec{x} .
$$

In 3D CB tomography, $i$ is usually a multi-index: at least one index, say $i_{1}$, is related to the source position on its trajectory, and an other index (or multi-index) $i_{2}$ is related to the detector pixel position in space at the source position $i_{1}$ or equivalently the direction of the ray from the source position at $t\left(i_{1}\right)$ (usually, in 3D CB, $i_{2}$ is a multi-index of two values because the direction space $S^{2}$ is a two dimensional set). Thus $t$ is a function of $i_{1}$ (the time depends on the source position), thus $d_{t, i}$ is $d_{t\left(i_{1}\right), i_{2}}$, where $i=\left(i_{1}, i_{2}\right)$, $i_{1} \in I_{1}, i_{2} \in I_{2}, I=I_{1} \times I_{2}$ and

$$
\begin{aligned}
d_{t\left(i_{1}\right), i_{2}} & =\mathcal{D} f_{\vec{\Gamma}_{t\left(i_{1}\right)}}\left(t\left(i_{1}\right), \vec{\zeta}\left(i_{2}\right)\right) \\
& =\sum_{j \in J}\left(\sum_{k \in K} B_{\left(i_{1}, i_{2}\right), k} \Gamma_{t\left(i_{1}\right)_{k, j}}\right) f_{j}
\end{aligned}
$$

Thus,

$$
\mathbf{d}_{\mathbf{i}_{1}}=\mathbf{B}_{\mathbf{i}_{1}} \boldsymbol{\Gamma}_{\mathbf{t}\left(\mathbf{i}_{1}\right)} \mathbf{f}, i_{1}=1, \ldots, n_{I_{1}}
$$


where the $n_{I_{1}}$ matrices $\mathbf{B}_{\mathbf{i}_{1}}$ are $n_{I_{2}} \times n_{K}\left(n_{I_{1}}\right.$ sets of $n_{I_{2}}$ lines of a classical ART matrix $\mathbf{B}$ corresponding to each $i_{1}$ source position, $\left.i_{1}=1, \ldots, n_{I_{1}}\right)$ and the matrices $\boldsymbol{\Gamma}_{\mathbf{t}\left(\mathbf{i}_{1}\right)}$ are $n_{K} \times n_{J}$ matrices coding the deformations by $\vec{\Gamma}_{t\left(i_{1}\right)}$ of the basis functions $e_{j}$ into the basis fuctions $b_{k}$, for each considered time $t\left(i_{1}\right), i_{1}=1, \ldots, n_{I_{1}}$. With this formulation, an algebraic matrix for dynamic tomography differs from the classical algebraic method only by the introduction of the matrices $\boldsymbol{\Gamma}_{\mathbf{t}\left(\mathbf{i}_{1}\right)}$. Just as for classical tomography, iterative methods are used for solving the linear system (10) and the matrices $\mathbf{B}$ and $\boldsymbol{\Gamma}_{\mathbf{t}\left(\mathbf{i}_{1}\right)}, i_{1}=1, \ldots, n_{I_{1}}$ are generally not stored but computed during the iterations.

\section{ANALYTIC DYNAMIC RECONSTRUCTION}

We recall briefly in this section a class of 3D deformations which can be analytically compensated in 3D Cone Beam reconstruction, see [23] and [16], [10] for 2D fan-beam dynamic tomography. In order to stay in the framework of 3D CB reconstruction, we consider the deformations $\vec{\Gamma}_{t}$ which transform the set of convergent acquisition lines at time $t$ into an other set of convergent lines at the reference time, i.e. we consider deformations that globally preserve the $\mathrm{CB}$ acquisition geometry. The deformation $\vec{\Gamma}_{t}: \mathbb{R}^{3} \rightarrow \mathbb{R}^{3}$ preserves the $\mathrm{CB}$ aquisition geometry globally if it transforms the source point $\vec{a}(t) \in \mathbb{R}^{3}$ at time $t$ into a virtual source point $\vec{\Gamma}_{t}(\vec{a}(t))$ at the reference time (e.g. $t=0$ as we assumed) and if any half line from $\vec{a}(t)$ is transformed into a virtual half line from the virtual source $\vec{\Gamma}_{t}(\vec{a}(t))$, see Fig. 2 . Let us use the spherical coordinates $(l, \vec{\zeta}) \in \mathbb{R}^{+} \times S^{2}$ of $\vec{x}-\vec{a}(t)$, i.e., $\vec{x}=\vec{a}(t)+l \vec{\zeta}$. The half line from $\vec{a}(t)$ in the direction $\vec{\zeta}$ is denoted by $\vec{a}(t)+\mathbb{R}^{+} \vec{\zeta}$. Assume $\vec{x}$ belongs to $\vec{a}(t)+\mathbb{R}^{+} \vec{\zeta} . \vec{\Gamma}_{t}$ leaves the CB geometry globally invariant if $\forall t \in T, \forall \vec{\zeta} \in S^{2}$,

$$
\vec{\Gamma}_{t}\left(\vec{a}(t)+\mathbb{R}^{+} \vec{\zeta}\right)=\vec{\Gamma}_{t}(\vec{a}(t))+\mathbb{R}^{+} \vec{\Gamma}_{S^{2}, t}(\vec{\zeta}),
$$

where $\vec{\Gamma}_{S^{2}, t} S^{2} \longrightarrow S^{2}$ is a diffeomorphism (bi-regular bijection) on the unit sphere which associates to a direction $\vec{\zeta}$ at $t$ a direction $\vec{\Gamma}_{S^{2}, t}(\vec{\zeta})$ at the reference time. More precisely, the following deformation leaves the 3D CB geometry globally invariant:

$$
\vec{\Gamma}_{t}(\vec{x})=\vec{\Gamma}_{t}(\vec{a}(t)+l \vec{\zeta})=\vec{\Gamma}_{t}(\vec{a}(t))+\Gamma_{t, \vec{\zeta}}(l) \vec{\Gamma}_{S^{2}, t}(\vec{\zeta}),
$$

where $\Gamma_{t, \vec{\zeta}}$ is a bi-regular bijective function on $\mathbb{R}^{+}$such that $\Gamma_{t, \vec{\zeta}}(0)=0$. In the following $\Gamma_{t, \vec{\zeta}}$ is linear in order to stay in the framework of the $3 \mathrm{D} \mathrm{CB}$ transform (more complex bijections would yield a non constant Jacobian in (16) which would lead to generalized CB transforms for which we do not have inversion formulas). Thus let $\Gamma_{t, \vec{\zeta}}(l)=c_{t, \vec{\zeta}} l$ with $c_{t, \vec{\zeta}}>0$ being both a function of $t$ but also of $\vec{\zeta}$. We then can write (12) as:

$$
\vec{\Gamma}_{t}(\vec{x})=\vec{\Gamma}_{t}(\vec{a}(t))+c_{t, \vec{\zeta}} l \vec{\Gamma}_{S^{2}, t}(\vec{\zeta})
$$

Now, let $\vec{v}(t) \stackrel{\text { def }}{=} \vec{\Gamma}_{t}(\vec{a}(t))-\vec{a}(t)$. We can decompose $\vec{\Gamma}_{t}$ of (13) into:

$$
\vec{\Gamma}_{t}=\vec{T}_{\vec{v}(t)} \circ \vec{\Delta}_{t}
$$

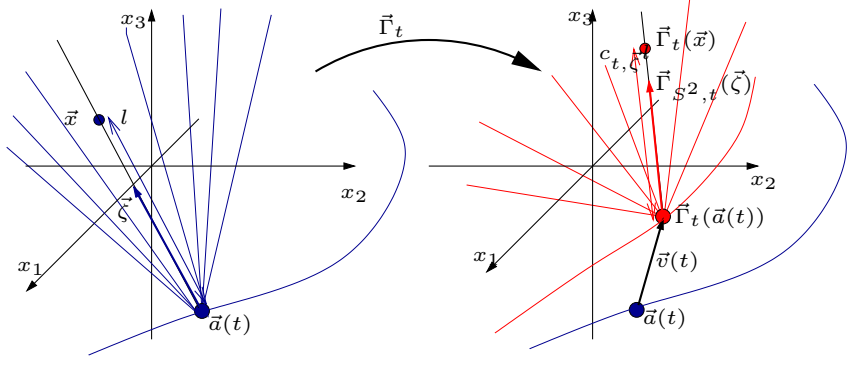

Fig. 2. Deformation $\vec{\Gamma}_{t}$ preserving the 3D CB geometry globally

where $\vec{T}_{\vec{v}(t)}$ is the translation of vector $\vec{v}(t)\left(\vec{T}_{\vec{v}(t)}(\vec{x})=\right.$ $\vec{v}(t)+\vec{x})$ and

$$
\vec{\Delta}_{t}(\vec{x})=\vec{a}(t)+c_{t, \vec{\zeta}} l \vec{\Gamma}_{S^{2}, t}(\vec{\zeta}) .
$$

We then remark that the deformation $\vec{\Delta}_{t}$ can be analytically compensated directly within each $3 \mathrm{D}$ projection at fixed $t$ :

$$
\begin{aligned}
\mathcal{D}_{t} f_{\vec{\Delta}_{t}}(\vec{\zeta}) & =\int_{0}^{+\infty} f\left(\vec{a}(t)+c_{t, \vec{\zeta}} l \vec{\Gamma}_{S^{2}, t}(\vec{\zeta})\right) d l \\
& =\frac{1}{c_{t, \vec{\zeta}}} \mathcal{D}_{t} f\left(\vec{\Gamma}_{S^{2}, t}(\vec{\zeta})\right) .
\end{aligned}
$$

Thus, from (16) we have

$$
\mathcal{D}_{t} f(\vec{\zeta})=c_{t, \vec{\Gamma}_{S^{2}, t}^{-1}(\vec{\zeta})} \mathcal{D}_{t} f_{\vec{\Delta}_{t}}\left(\vec{\Gamma}_{S^{2}, t}^{-1}(\vec{\zeta})\right) .
$$

Combining now (14) and (17) we have

$$
\mathcal{D}_{t} f_{\vec{T}_{\vec{v}(t)}}(\vec{\zeta})=c_{t, \vec{\Gamma}_{S^{2}, t}^{-1}(\vec{\zeta})} \mathcal{D}_{t} f_{\vec{T}_{\vec{v}(t)} \circ \vec{\Delta}_{t}}\left(\vec{\Gamma}_{S^{2}, t}^{-1}(\vec{\zeta})\right) .
$$

The deformation $\vec{\Delta}_{t}$ can thus be very simply compensated within the projection $\mathcal{D}_{t} f_{\vec{T}_{\vec{v}(t)} \circ \vec{\Delta}_{t}}$, i.e. within $\mathcal{D}_{t} f_{\vec{\Gamma}_{t}}$, in order to compute $\mathcal{D}_{t} f_{\vec{T}_{\vec{v}(t)}}$. Afterwards the translation $\vec{T}_{\vec{v}(t)}$ can be simply compensated: instead of reconstructing $f$ from the 3D CB acquisition on the real trajectory $\vec{a}(t), t \in T \subset$ $\mathbb{R}$, we have to perform the reconstruction from the virtual trajectory $\vec{\Gamma}_{t}(\vec{a}(t))=\vec{a}(t)+\vec{v}(t), t \in T \subset \mathbb{R}$. Indeed, from the definition (1)

$$
\begin{aligned}
\mathcal{D}_{t} f_{\vec{T}_{\vec{v}(t)}}(\vec{\zeta}) & =\int_{0}^{+\infty} f(\vec{a}(t)+\vec{v}(t)+l \vec{\zeta}) d l \\
& =\int_{0}^{+\infty} f\left(\vec{\Gamma}_{t}(\vec{a}(t))+l \vec{\zeta}\right) d l .
\end{aligned}
$$

Thus, the Tuy-Grangeat stable reconstruction conditions [24], [25] at a point $\vec{x}$ will be read on the virtual trajectory $\vec{\Gamma}_{t}(\vec{a}(t))$, just as the possibility to use modern $3 \mathrm{D} \mathrm{CB}$ reconstruction algorithms [26], [7], [27].

\section{NUMERICAL EXPERIMENTS}

A realistic digital phantom of the thorax was used to evaluate the behavior of different reconstruction methods in the presence of respiratory motion. Lung radiotherapy optimization is a typical application for this study. The phantom was obtained from a 4D CT image acquired on a scanner synchronised with a respiratory signal [28]. One 
of its ten 3D CT images was chosen to represent $f$. Nine dense motion vector fields were computed by deformable registration between each 3D CT image and the reference. From them, continuous trajectories for each voxel of $f$ were obtained by supposing that the motion is linear between two consecutive respiratory instants. A set of cone-beam projections $g_{\mathcal{D}}$ were computed using a projector taking into account this motion model and the geometry of an existing cone-beam CT scanner. Fig. 3 allows the comparison of the reference $\mathrm{CT}$ image with different reconstructions.

Motion artifacts, such as blur and streaks, are clearly visible, both with Feldkamp's analytic method [29] and with Simultaneous Algebraic Reconstruction Technique (SART) [30], which suppose that the patient remained static during the acquisition. The motion does not globally preserve the cone-beam geometry as described in part III, that is why only a heuristic motion compensation during the backprojection step of Feldkamp's algorithm was feasible [31]. The blur is reduced but streaks are still visible, particularly on the axial slice, because the compensation is not exact. Finally, motion compensated SART with the projector used for the computation of the cone-beam projections fully eliminate the motion artifacts.

\section{CONCLUSIONS AND FUTURE WORKS}

We have presented a framework for both analytic and algebraic method in dynamic tomography with known time dependent deformations of the space. We have provided numerical experiments showing that algebraic methods can compensate the respiratory motion in 4D CT from acquisition during lung radiotherapy applications. In future works, we will compare analytic and algebraic approaches on this application.

\section{REFERENCES}

[1] M. Grass, R. Manzke, T. Nielsen, P. Koken, R. Proksa, M. Natanzon, and G. Shechter, "Helical cardiac cone beam reconstruction using retrospective ECG gating," Phys. Med. Biol., vol. 48, pp. 3069-3083, September 2003.

[2] E. C. Ford, G. S. Mageras, E. Yorke, and C. C. Ling, "Respiratorycorrelated spiral CT: a method of measuring respiratory-induced anatomic motion for radiation treatment planning," Medical Physics, vol. 30(1), pp. 88-97, January 2003.

[3] P. Grangeat, "Mathematical framework of cone beam 3D reconstruction via the first derivative of the Radon transform," Mathematical Methods in Tomography, G.T. Herman, A.K. Louis, F. Natterer, Lecture Notes in Mathematics, pp. 66-97, 1991.

[4] M. Defrise, R. Clack, and D. Townsend, "The solution to the 3D image reconstruction problem from 2D parallel projections," J. Opt. Soc. Am. A, vol. 10, pp. 869-877, 1993.

[5] A. Katsevich, "Analysis of an exact inversion algorithm for spiral cone beam CT," Phys. Med. Biol., vol. 47, pp. 2583-98, 2002.

[6] — , "Theoretically exact filtered back-projection type inversion algorithm for spiral CT," SIAM. J. Appl. Math., vol. 62, pp. 201226, 2002.

[7] J. Pack and F. Noo, "Cone-beam reconstruction using 1d filtering along the projection of m-lines," Inverse Problems, vol. 21, no. 3, pp. 1105$1120,2005$.

[8] Y. Zou and X. Pan, "Image reconstruction on PI-lines by use of filtered backprojection in helical cone-beam CT," Phys. Med. Biol., vol. 49, pp. 2717-2731, 2004.

[9] C. Crawford, K. King, C. Ritchie, and J. Godwin, "Respiratory compensation in projection imaging using a magnification and displacement model," IEEE Transactions on Medical Imaging, vol. 15, pp. $327-332,1996$
[10] S. Roux, L. Desbat, A. Koenig, and P. Grangeat, "Exact reconstruction in 2D dynamic CT: compensation of time-dependent affine deformations," Phys. Med. Biol., vol. 49(11), pp. 2169-82, June 2004.

[11] R. Fulton, B. Hutton, M. Braun, B. Ardekani, and R. Larkin, "Use of 3D reconstruction to correct for patient motion in SPECT," Physics in Medicine and Biology, vol. 39, no. 3, pp. 563-574, Mar 1994.

[12] B. Hutton, A. Kyme, Y. Lau, D. Skerrett, and R. Fulton, "A hybrid 3-D reconstruction/registration algorithm for correction of head motion in emission tomography," IEEE Transactions on Nuclear Science, vol. 49, no. 1, pp. 188-194, Feb. 2002.

[13] B. Feng, H. Gifford, R. Beach, G. Boening, M. Gennert, and M. King, "Use of three-dimensional Gaussian interpolation in the projector/backprojector pair of iterative reconstruction for compensation of known rigid-body motion in SPECT," IEEE Transactions on Medical Imaging, vol. 25, no. 7, pp. 838-844, Jul 2006.

[14] L. Livieratos, L. Stegger, P. Bloomfield, K. Schafers, D. Bailey, and P. Camici, "Rigid-body transformation of list-mode projection data for respiratory motion correction in cardiac PET," Physics in Medicine and Biology, vol. 50, no. 14, pp. 3313-3322, Jul 2005. [Online]. Available: http://dx.doi.org/10.1088/0031-9155/50/14/008

[15] F. Lamare, T. Cresson, J. Savean, C. Cheze Le Rest, A. Reader, and D. Visvikis, "Respiratory motion correction for PET oncology applications using affine transformation of list mode data," Physics in Medicine and Biology, vol. 52, no. 1, pp. 121-140, Jan 2007. [Online]. Available: http://dx.doi.org/10.1088/0031-9155/52/1/009

[16] L. Desbat, S. Roux, and P. Grangeat, "Compensation of some time dependent deformations in tomography," IEEE transaction on Medical Imaging, vol. 26, no. 2, pp. 261-269, 2007.

[17] L. Desbat, M. Fleute, M. Defrise, X. Liu, C. Huberson, R. Laouar, R. Martin, J. Guillou, and S. Lavallée, "Minimally Invasive Interventional Imaging for Computer Assisted Orthopedic Surgery," in SURGETICA'2002. Sauramps médical, 2002, pp. 288-295, (best conference paper).

[18] K. Wiesent, K. Barth, N. Navab, P. Durlak, T. Brunner, O. Schuetz, and W. Seissler, "Enhanced 3-d-reconstruction algorithm for c-arm systems suitable for interventional procedures," IEEE Trans. Med. Imaging, vol. 19, no. 5, pp. 391-403, 2000.

[19] C. Blondel, R. Vaillant, G. Malandain, and N. Ayache, "3D tomographic reconstruction of coronary arteries using a precomputed $4 \mathrm{D}$ motion field," Physics in Medicine and Biology, vol. 49, no. 11, pp. 2197-2208, 2004.

[20] C. Blondel, G. Malandain, R. Vaillant, and N. Ayache, "Reconstruction of coronary arteries from a single rotational X-ray projection sequence," IEEE Transactions on Medical Imaging, vol. 25, no. 5, pp. 653-663, 2006.

[21] M. Reyes, G. Malandain, P. Koulibaly, M. González Ballester, and J. Darcourt, "Respiratory motion correction in emission tomography image reconstruction," in Medical Image Computing and ComputerAssisted Intervention (MICCAI2005), vol. 8, no. Pt 2, 2005, pp. 369376.

[22] M. Reyes, G. Malandain, P. Koulibaly, and J. Darcourt, "Respiratory motion correction in emission tomography imaging," in Eighth International Meeting on Fully Three-dimensional Image Reconstruction in Radiology and Nuclear Medicine, Salt Lake City, USA, 2005.

[23] L. Desbat, S. Roux, and P. Grangeat, "Compensation de déformations en tomographie dynamique 3D conique," Traitement du Signal, vol. 23, no. 6 .

[24] H. Tuy, "An inversion formula for cone-beam reconstruction," SIAM J. Appl. Math., vol. 43, no. 3, pp. 546-552, 1983.

[25] P. Grangeat, "Analyse d'un sytème d'imagerie 3d par reconstructions à partir de radiographies $x$ en géométrie conique." Ph.D. dissertation, ENST, 1987.

[26] A. Katsevich, "A general scheme for contructing inversion algorithms for cone beam CT," International journal of Mathematics and Mathematical Sciences, vol. 21, pp. 1305-1321, 2003.

[27] E. Sidky and X. Pan, "A minimum data FBP-type algorithm for image reconstruction in cone-beam CT," in Fully $3 D$ image reconstruction in radiology and medicine proceeding, Salt Lake City, 2005, pp. 291294.

[28] J. Vandemeulebroucke, D. Sarrut, and P. Clarysse, "Point-validated pixel-based breathing thorax model," in Proceedings of the International Conference on the Use of Computers in Radiation Therapy ICCR'2007, 2007.

[29] L. Feldkamp, L. Davis, and J. Kress, "Practical cone-beam algorithm," 


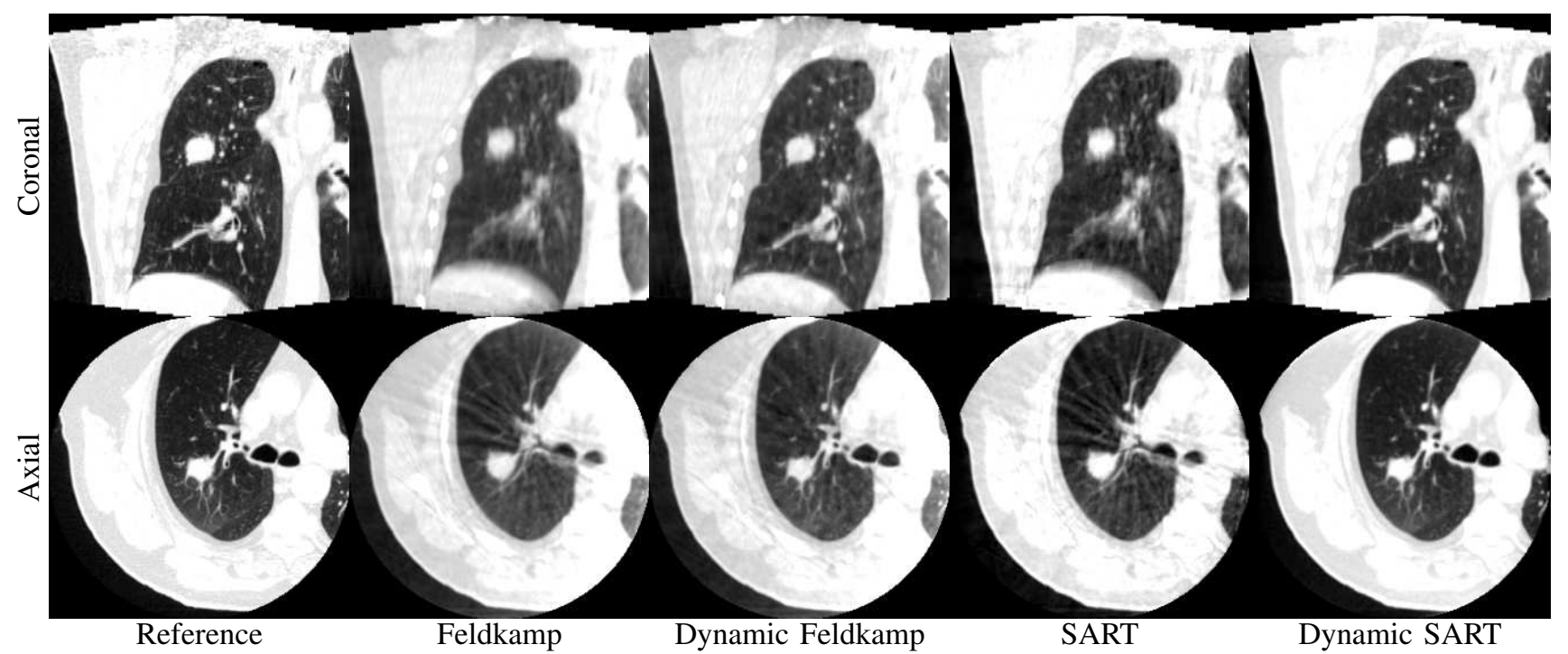

Fig. 3. Coronal and axial slices of the reconstruction results on the realistic digital phantom of the thorax. Feldkamp and SART methods do not take into account the motion. Dynamic Feldkamp uses a heuristic compensation of the motion during the backprojection step of Feldkamp algorithm. Dynamic SART is identical to the SART method with the projector taking into account the motion.

Journal of Optical Society of America A, vol. 1, no. 6, pp. 612-619, 1984.

[30] A. Andersen and A. Kak, "Simultaneous algebraic reconstruction technique (SART): a superior implementation of the art algorithm." Ultrason Imaging, vol. 6, no. 1, pp. 81-94, Jan 1984.

[31] C. Ritchie, C. Crawford, J. Godwin, K. King, and Y. Kim, "Correction of computed tomography motion artifacts using pixel-specific backprojection," IEEE Transactions on Medical Imaging, vol. 15, no. 3, pp. 333-342, 1996. 